\title{
Assessment of Erythropoietin Efficacy in Treatment of Anemia in Pre-dialysis Chronic Kidney Disease Patients Mostafa Fawzy Mohamed Selim*, ${ }^{1}$ Essam Eldin Mahmoud Lotfy', Lamiaa Abd Elwahab Mohamed ${ }^{2}$, Mahmoud Hosny Zahran ${ }^{1}$
}

Departments ${ }^{1}$ Internal Medicine \& ${ }^{2}$ Clinical Pathology, Faculty of Medicine, Zagazig University, Zagazig, Egypt. *Corresponding author: Mostafa Fawzy Mohamed Selim, Mobile : (+20)01558527334, Email: mostafaselim945@yahoo.com

\begin{abstract}
Background: Anemia is the most frequent condition in patients suffering from chronic renal disease. Iron supplements are commonly prescribed for these patients using oral and intravenous iron with or without erythropoietin therapy.

Objectives: This study aimed at comparing the efficacy of IV iron versus oral iron in raising hemoglobin levels in predialysis chronic kidney disease (CKD) patients.

Patients and Methods: A prospective cohort study that was conducted in Met-Ghamr Hospital of Nephrology and Urology from February 2019 to July 2019. The study included 50 CKD patients with anemia in the pre-dialysis stage. Group 1 consisted of 25 patients that were given IV iron while the 25 patients of group 2 were given oral iron. Both groups were monitored for 4 months for changes in hemoglobin levels and side effects. After the initial 4 months, patients in both groups who did not have a sufficient hemoglobin response to iron supplementation were prescribed an additional erythropoiesis-stimulating agent (ESA) with monitoring of hemoglobin response for the consequent 2 months.

Results: We found a significant difference in the hemoglobin response between the two sets of patients in favor of the IV route after 4 months. We also found the safety profile of both routes to be comparable. After 4 months, the patients that received additional dose of Epoetin alfa (4000 IU/week for 2 months) showed a significant rise in hemoglobin level over the next 2 months compared to patients who did not receive the additional dose.

Conclusion: We concluded that intravenous iron supplement is better than oral iron supplement in correction of anemia in pre-dialysis patients.
\end{abstract}

Keywords: Erythropoietin, Chronic Kidney Disease, Glomerular Filtration Rate, Anemia, Iron.

\section{INTRODUCTION}

Chronic kidney disease (CKD) is defined as kidney damage or an estimated glomerular filtration rate (eGFR) less than $60 \mathrm{ml} / \mathrm{min} / 1.73 \mathrm{~m}$ persisting for three months or more irrespective of the cause.Kidney damage can be demonstrated through urine analysis, imaging or renal biopsy ${ }^{(1)}$.

CKD is classified based on the GFR into 5 stages from one to five. Causes of CKD vary from diabetes mellitus, hypertension (the two most common causes), recurrent renal stones and glomerular diseases ${ }^{(2)}$. The presentation of CKD can vary from being asymptomatic to producing symptoms such as nausea, vomiting, hiccough, hypertension, pruritus and uremic encephalopathy ${ }^{(2)}$.

CKD has various complications such as cardiovascular complications (the most common cause of mortality), chronic kidney disease-mineral bone disease (CKDMBD), dyslipidemia and malnutrition. However, anemia is the most common complication of CKD. Anemia is associated with progression of CKD, poor quality of life and increased morbidity and mortality ${ }^{(3)}$.

Anemia is defined as hemoglobin $(\mathrm{Hb})<13 \mathrm{~g} / \mathrm{dl}$ in adult male and $<12 \mathrm{~g} / \mathrm{dl}$ in adult female. The mechanism of anemia in CKD is multifactorial: relative erythropoietin deficiency from reduced renal mass, iron and nutritional deficiencies and various pro-inflammatory mediators commonly elevated in CKD that may affect the erythropoiesis ${ }^{(4)}$. Iron deficiency is a common reversible factor contributing to the development of anemia in CKD patients and can be easily treated. Guidelines have a consensus about iron deficiency anemic hemodialysis patients to be supplied with iron supplementation through the IV route. However, there is no recommendation yet on the most appropriate for patients not yet on dialysis ${ }^{(5)}$.

Three routes of administration for iron are available: oral, intramuscular, and intravenous. Moreover, iron deficiency is the most common cause of resistance to erythropoietin therapy, contributing to ineffective erythropoiesis and hematocrit/haemoglobin values below the recommended target range of g/dL-12 g/dL (33\% $36 \%)$. Iron supplementation improves iron indices and haemoglobin concentration and reduces the required ESA dose ${ }^{(6)}$.

Most of the randomized controlled trials have compared intravenous (IV) iron to oral iron in nondialysis-dependent CKD patients. Transferrin saturation (TSAT), serum ferritin and haemoglobin levels are useful markers of iron deficiency. In our population, most of the people had nutritional anemia adding to the anemia of 
CKD. To replenish a good amount of iron, supplements are used by means of oral or IV therapy ${ }^{(7)}$.

The aim of this work was comparing the efficacy of IV iron versus oral iron in raising hemoglobin levels in predialysis chronic kidney disease (CKD) patients.

\section{PATIENTS AND METHODS}

A prospective study was conducted in Met-Ghamr Hospital of Nephrology and Urology from February 2019 to July 2019. The study included 50 chronic kidney disease (CKD) patients with anemia in the pre-dialysis stage with Glomerular filtration rate (GFR) falling in the region of stage 4 to stage $5 \mathrm{CKD}$ that were divided into two equal groups. Each group contained 8 males and 17 females with a mean age of $57.12 \pm 17.72$ years.

\section{Ethical and patients' approval:}

Ethical approval was taken from the Ethical Clearance Committee of Zagazig University and verbal consent was taken from all the patients. The work has been carried in accordance with the Code of Ethics of the World Medical Association (Declaration of Helsiniki) for studies involving humans.

Inclusion criteria: CKD Patients (either on hemodialysis or in pre-dialysis stage) with hemoglobin level $\leq 11 \mathrm{gm} / \mathrm{dl}$ either using erythropoietin or not.

Exclusion criteria: Patients with hemoglobin level higher than $11 \mathrm{gm} / \mathrm{dl}$. Patients with macrocytic anemia attributed to folate or vitamin B12 deficiency. Patients who had kidney transplantation. Patients who had blood transfusions within the last three months. Patients with acute or chronic bleeding. Patients with active infection. Patients with malignant disease. Patients with advanced cardiovascular disease. Pregnant or lactating female patients.

The patients were allocated into two groups, 25 patients in each group to receive oral iron or IV sucrose. In the oral iron group, ferrous fumarate $(150 \mathrm{mg}=47 \mathrm{mg}$ elemental iron) was given thrice a day and in the IV iron group $100 \mathrm{mg}$ of iron sucrose.

In order to minimize the number of dosage adjustments and to make the comparison between hemoglobin responses in both groups more clear, no erythropoiesis stimulating agent (ESA) were given to patients who never received it before for the first 4 months and no ESA dose changes for patients who were already on it for the first 4 months. Hemoglobin level were followed up at the $2^{\text {nd }}$ month, $4^{\text {th }}$ month and the $6^{\text {th }}$ month. After 4 months, cases who had insufficient hemoglobin response (we arbitrarily defined it as failure to achieve values above $10 \mathrm{~g} / \mathrm{dl}$ ) were given an ESA in the form of 4000 IU Epoetin alfa subcutaneously once weekly. Patients who were previously on ESAs were given an additional 4000 IU Epoetin alfa subcutaneously once weekly (i.e the total dose was twice weekly).

\section{Statistical Analysis}

All data were collected, tabulated and statistically analyzed using SPSS version 25.0 for windows (SPSS Inc., Chicago, IL, USA). Quantitative data were expressed as the mean \pm SD and range (if normally distributed) \& as median and inter-quartile range (IQR) (if not normally distributed), and qualitative data were expressed as absolute frequencies "number" \& relative frequencies (percentage). Continuous data were checked for normality by using Shapiro Walk test.

Independent Student t-test was used to compare two groups of normally distributed data. Percent of categorical variables were compared using Chi-square test. All tests were two sided. P-value $\leq 0.05$ was considered statistically significant, $\mathrm{p}<0.001$ was considered highly statistically significant and $p>0.05$ was considered none statistically significant.

\section{RESULT}

Table (1) showed that there was no significant difference in the demographic characteristics between the two groups.

Table (2) showed that hypertension and diabetes mellitus were the most common causes of CKD across all our groups. There was no significant difference in characteristics of CKD etiology.

Table (3) showed significant difference in hemoglobin rise in favor of the IV iron group.

Figure (1) showed significant difference in hemoglobin rise in favor of patients who received ESAs.

Table (4) showed that there was higher GFR level at baseline, which was associated with higher hemoglobin levels at the end of the study.

Table (5) showed that higher GFR level at baseline was associated with higher hemoglobin levels at the end of the study. ESAs use was not associated with decreased GFR and progression of the kidney disease.

Table (6) showed that higher GFR level at baseline was associated with higher hemoglobin levels at the end of the study. There was non-significant weak negative correlation between baseline PTH level and hemoglobin levels at the end of the study. 
Table (1): Distribution of the demographic characteristics of group

\begin{tabular}{|c|c|c|c|c|c|}
\hline & & \multicolumn{4}{|c|}{ Groups } \\
\hline & & \multicolumn{2}{|c|}{ (1) $(n=25)$} & \multicolumn{2}{|c|}{ (2) $(n=25)$} \\
\hline & & No. & $\%$ & No. & $\%$ \\
\hline \multirow{3}{*}{ Sex } & Male & 8 & 32 & 13 & 52 \\
\hline & Female & 17 & 68 & 12 & 48 \\
\hline & ${ }^{c 2} p$ & \multicolumn{4}{|c|}{$\mathrm{p}_{1}=0.152$} \\
\hline \multirow{3}{*}{ Age (years) } & Min. - Max. & \multicolumn{2}{|c|}{$24.0-85.0$} & \multicolumn{2}{|c|}{$24.0-77.0$} \\
\hline & Mean \pm SD. & \multicolumn{2}{|c|}{$57.12 \pm 17.72$} & \multicolumn{2}{|c|}{$50.96 \pm 14.12$} \\
\hline & ${ }^{t} \mathbf{p}$ & \multicolumn{4}{|c|}{$\mathrm{p}_{1}=0.180$} \\
\hline
\end{tabular}

$\square$ : $p$ value for Chi square test $\quad$ tp: $p$ value for Student $t$-test, $\mathrm{p}_{1}: \mathrm{p}$ value for comparing between group 1 and group 2

Table (2): Distribution of the studied groups according to the cause of CKD

\begin{tabular}{|c|c|c|c|c|}
\hline \multirow{3}{*}{ Cause of CKD } & \multicolumn{4}{|c|}{ Group } \\
\hline & \multicolumn{2}{|c|}{ (1) $(n=25)$} & \multicolumn{2}{|c|}{ (2) $(n=25)$} \\
\hline & No. & $\%$ & No. & $\%$ \\
\hline HTN & 13 & 52.0 & 12 & 48.0 \\
\hline$P$ & \multicolumn{4}{|c|}{$p_{1}=0.777$} \\
\hline $\mathbf{D M}$ & 8 & 32.0 & 8 & 32.0 \\
\hline$P$ & \multicolumn{4}{|c|}{$p_{1}=1.000$} \\
\hline Unknown & 1 & 4.0 & 1 & 4.0 \\
\hline $\boldsymbol{P}$ & \multicolumn{4}{|c|}{$\mathrm{FE}_{\mathrm{p}_{1}=1.000}$} \\
\hline Glomerulonephritis & 1 & 4.0 & 4 & $\mathbf{1 6 . 0}$ \\
\hline$P$ & \multicolumn{4}{|c|}{$\mathrm{FE}_{\mathrm{p}_{1}=0.349}$} \\
\hline Pregnancy complication & 2 & 8.0 & $\mathbf{0}$ & 0.0 \\
\hline $\boldsymbol{P}$ & \multicolumn{4}{|c|}{${ }^{\mathrm{FE}} \mathrm{p}_{\mathbf{1}}=0.490$} \\
\hline ADPKD & $\mathbf{0}$ & 0.0 & 2 & 8.0 \\
\hline$P$ & \multicolumn{4}{|c|}{$\mathrm{FE}_{\mathrm{p}_{1}=0.490}$} \\
\hline Analgesic nephropathy & 1 & 4.0 & 1 & 4.0 \\
\hline $\boldsymbol{P}$ & \multicolumn{4}{|c|}{${ }^{F E} \mathbf{p}_{1}=1.000$} \\
\hline Obstructive uropathy & $\mathbf{0}$ & 0.0 & $\mathbf{0}$ & 0.0 \\
\hline $\boldsymbol{P}$ & \multicolumn{4}{|c|}{${ }^{F E} p_{1}=0.667$} \\
\hline SLE & $\mathbf{1}$ & 4.0 & 1 & 4.0 \\
\hline $\boldsymbol{P}$ & \multicolumn{4}{|c|}{${ }^{\mathrm{FE}} \mathbf{p}_{1}=1.000$} \\
\hline Chronic pyelonephritis & $\mathbf{1}$ & 4.0 & $\mathbf{0}$ & $\mathbf{0 . 0}$ \\
\hline $\boldsymbol{P}$ & \multicolumn{4}{|c|}{${ }^{F E} p_{1}=1.000$} \\
\hline
\end{tabular}

$\mathrm{p}$ : $\mathrm{p}$ value for Chi square test $\quad{ }^{\mathrm{FE}} \mathrm{p}$ : $\mathrm{p}$ value for Fisher Exact

$\mathrm{p}_{1}$ : $\mathrm{p}$ value for comparing between group IA and group IB

Table (3): Comparison between hemoglobin rises in the two groups after 4 months (group 1 was on oral iron and group 2 was on IV iron)

\begin{tabular}{|c|c|c|}
\hline \multirow{2}{*}{$\begin{array}{l}\text { Hemoglobin/Increase } \\
\text { Till } 4^{\text {th }} \text { month }\end{array}$} & \multicolumn{2}{|c|}{ Group } \\
\hline & (1) $(n=25)$ & (2) $(\mathrm{n}=25)$ \\
\hline Min. - Max. & $-1.0-1.30$ & $-2.0-1.90$ \\
\hline Median (IQR) & $0.10(-0.05-0.60)$ & $0.80(0.0-1.25)$ \\
\hline 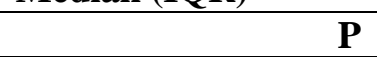 & \multicolumn{2}{|c|}{$0.049^{*}$} \\
\hline
\end{tabular}

p: $\mathrm{p}$ value for Mann Whitney test for comparing between group 1and group 2. *: Statistically significant at $\mathrm{p} \leq 0.05$ 


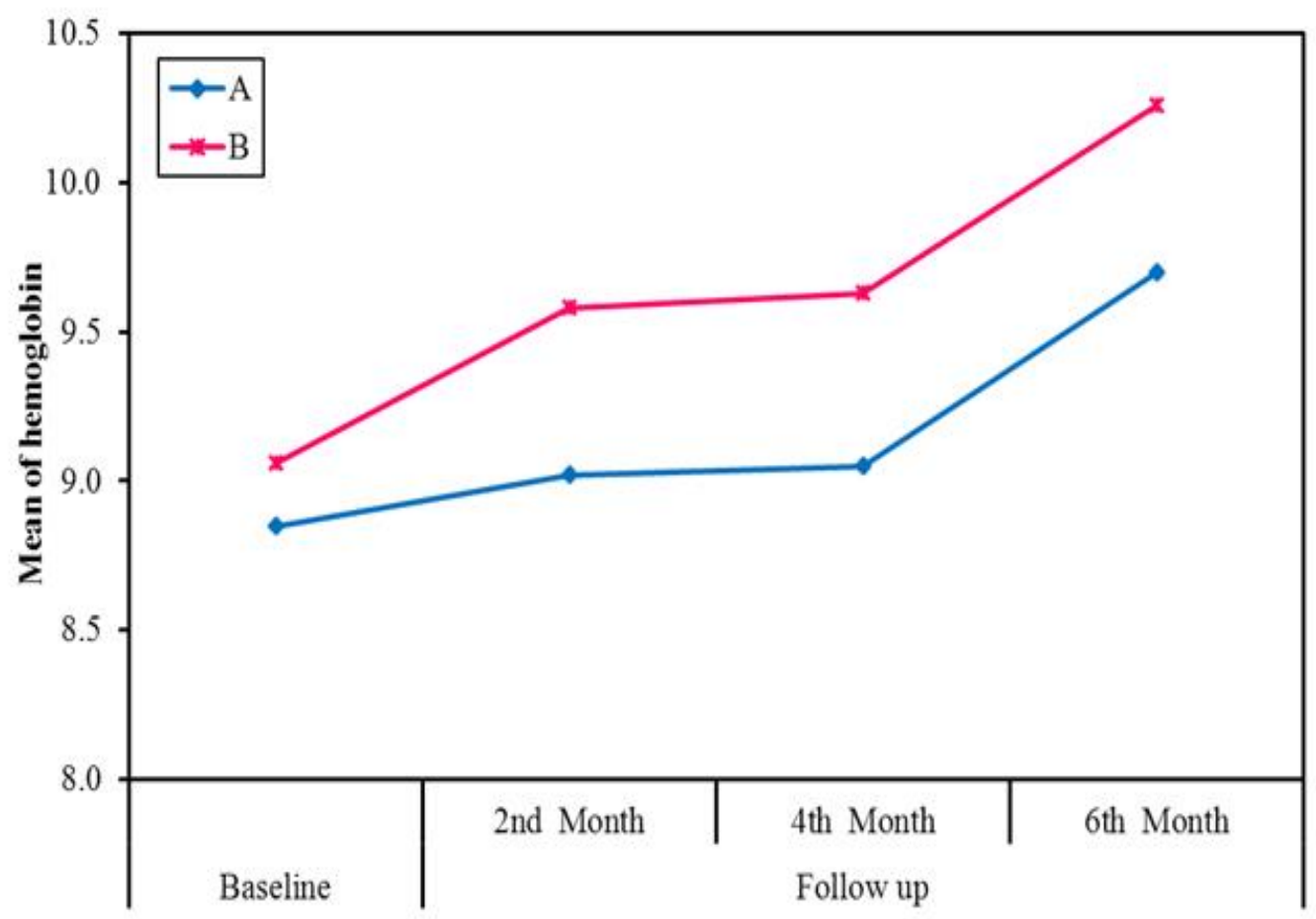

A: group 1.

B: group 2.

Figure (1): Follow up of hemoglobin levels in group during the study

Table (4): Correlation between hemoglobin rise at the end of the study and baseline GFR

\begin{tabular}{|l|l|c|}
\hline \multicolumn{2}{|c|}{} & Hemoglobin rise after 6 months \\
\hline \multirow{2}{*}{ GFR } & $r_{s}$ & 0.427 \\
\cline { 2 - 3 } & $P$ & 0.033 \\
\hline
\end{tabular}

$\mathrm{r}_{\mathrm{s}}$ : Spearman coefficient

*: Statistically significant at $\mathrm{p} \leq 0.05$

Table (5): The relation between ESAs use and the GFR at the end of the study

\begin{tabular}{|c|c|c|}
\hline \multirow{2}{*}{ GFR decrease till $6^{\text {th }}$ month } & \multicolumn{2}{|c|}{ Received ESA after 4 months } \\
\hline & $\begin{array}{c}\text { No } \\
(n=22)\end{array}$ & $\begin{array}{c}\text { Yes } \\
(n=28)\end{array}$ \\
\hline Min. - Max. & $6.83-35.35$ & $5.50-36.36$ \\
\hline Median (IQR) & $12.73(11.1-16.9)$ & $14.40(12.5-23.9)$ \\
\hline$p$ & \multicolumn{2}{|c|}{0.143} \\
\hline
\end{tabular}

$\mathrm{p}: \mathrm{p}$ value for Mann Whitney test for comparing between no and yes

*: Statistically significant at $\mathrm{p} \leq 0.05$

Table (6): Correlation between hemoglobin rise at the end of the study and baseline iPTH level

\begin{tabular}{|c|c|c|c|}
\hline & & \multicolumn{2}{|c|}{ Hemoglobin till 6 $^{\text {th }}$ month } \\
\hline & & Group 1 & Group 2 \\
\hline \multirow{2}{*}{ PTH } & $\boldsymbol{r}_{s}$ & -0.124 & -0.272 \\
\cline { 2 - 4 } & $\boldsymbol{p}$ & 0.553 & 0.189 \\
\hline
\end{tabular}

$\mathrm{r}_{\mathrm{s}}$ : Spearman coefficient

*: Statistically significant at $\mathrm{p} \leq 0.05$ 


\section{DISCUSSION}

Our study group included 50 pre-dialysis patients that were divided into two groups, group (1) and group (2). Group 1 included 25 anemic pre dialysis (stage 4 and stage 5) CKD patients with a mean GFR of $14.12 \pm 7.94$, $68 \%$ of them were females (17 patients) while $32 \%$ of them were males (8 patients). The mean age was $57.12 \pm$ 17.72 years old. The mean TSAT at baseline was $15.08 \pm$ 4.73 while the mean ferritin at baseline was $102.60 \pm$ $46.98 \mathrm{ng} / \mathrm{ml}$. Group 2 included 25 anemic pre dialysis (stage 4 and stage 5) CKD patients with a mean GFR of $17.26 \pm 7.37,52 \%$ of them were males (13 patients) while $48 \%$ of them were females (12 patients). The mean age was $50.96 \pm 14.12$ years old. The mean TSAT at baseline was $16.44 \pm 5.94$, while the mean ferritin at baseline was $103.12 \pm 48.15$. There was no significant difference between the baseline characteristics of both groups.

At baseline, only 6 patients in both groups were already on erythropoiesis stimulating agents (ESAs), 4 patients of them were in group $1 \mathrm{~A}$ and 2 patients in group 1B. This falls in line with the fact that iron is usually the main treatment for anemia in the pre-dialysis CKD population while it is more of an adjuvant to ESAs in the anemic CKD population on dialysis ${ }^{(\mathbf{8})}$.

This study compared the efficacy of intravenous iron versus oral iron in the anemic pre-dialysis CKD population. Both groups were monitored for 4 months. The primary outcome was the change in hemoglobin concentration. The secondary outcome was the safety considerations and possible adverse effects that may accompany both lines of treatment. In order to allow a direct and clear comparison between the efficacies of intravenous iron versus oral iron, we kept patients who were already on ESAs on their same baseline ESA dose while avoiding initiating ESAs therapy in patients who never received it before. After 4 months, when we compared the increase in hemoglobin level between group 1 and group 2, we found a significant difference between the response of the two groups $(\mathrm{P}=0.049)$ pointing towards the superiority of intravenous iron in raising hemoglobin level. Our findings are consistent with the findings of Shepshelovich et al. ${ }^{(5)}$ who conducted a systematic review and a meta-analysis that included five trials that reported the number of pre-dialysis anemic CKD patients whose hemoglobin level response exceeded $1 \mathrm{~g} / \mathrm{dl}$ following supplementing them with iron through either of the oral or the intravenous routes. The $\mathbf{R R}$ (relative risk) of percentage of patients reaching a hemoglobin response of more than $1 \mathrm{~g} / \mathrm{dl}$ with intravenous iron compared to oral iron was $\mathbf{1 . 6 1}$ (95\% Confidence Interval, 1.39-1.87).

We found no evidence of fatal hypotension or of allergic reactions in patients receiving intravenous iron sucrose. GIT manifestations like (constipation, diarrhea, nausea, vomiting and dyspepsia) were more associated with oral iron. However, the GIT manifestations were generally mild and tolerable. There was no significant difference in rates of any side effects between the studied groups. These findings come in agreement with a metaanalysis by Avni et al. ${ }^{(9)}$ which showed that intravenous iron has a safety profile comparable to oral iron. Another study by Wang et al. ${ }^{(10)}$ found that the anaphylaxis risk for non-dextran iron products to be very low. However, these findings suggested that the higher efficacy and the comparable safety of intravenous iron should be interpreted cautiously telling that most of the studies comparing intravenous and oral iron were of short duration. Another factor to make us cautious about results is the questionable compliance of patients in the oral group due to the possible GIT side effects they may experience.

In this study, we used an intravenous iron sucrose compound with a price in the Egyptian market of about 20 EGP (about 1.25 \$). Given that we gave the patients one ampoule weekly for 4 months, the total cost of the intravenous iron treatment for each patient for the 4 months could be estimated to be about 320 EGP ( 20 \$). On the other hand, we gave the oral iron group an oral compound of a price of 6 EGP $(0.375 \$)$. Given that we gave the patients 3 caps per day, the total cost of the oral iron treatment for each patient for the 4 months could be estimated to be about 72 EGP (4.5 \$). Accordingly, intravenous iron is far more expensive than oral iron. However, comparing the cost-effectiveness of the two methods is more complex than just comparing the actual costs and there are other factors that should be taken into consideration when assessing this issue like the probable superior hemoglobin response with intravenous iron, delaying or decreasing the need for ESAs use, less GIT disturbance and possible better impact on patients' quality of life.

After the initial 4 months, patients in both groups who did not have a sufficient hemoglobin response to iron supplementation were prescribed an erythropoiesisstimulating agent. We arbitrarily defined the sufficient hemoglobin response as reaching a hemoglobin level above the $10 \mathrm{~g} / \mathrm{dl}$ barrier. Consequently, Epoetin alfa $4000 \mathrm{IU}$ was prescribed to 16 patients in group 1 and to 12 patients in group 2.

18 patients did not have a sufficient response after 4 months, distributed as 16 patients in group 1 and 2 patients in group 2. We monitored the hemoglobin response of all group I patients (prescribed additional ESAs or not) after 2 months. These 2 months coincided with the period from the $4^{\text {th }}$ to the $6^{\text {th }}$ months of our study. The results showed a significant difference between the patients who received Epoetin alfa and those who did not $(\mathrm{p}=0.001)$. In a systematic review by Cody and Hodson (11) comparing the effect of using ESAs versus no treatment or placebo in pre-dialysis patients, they reported 
significant increase in hemoglobin in ESAs-treated patients compared to placebo or no treatment patients (MD $1.90 \mathrm{gm} / \mathrm{L}, 95 \%$ CI -2.34 to -1.47 ).

These results also showed a direct relation between the severity of the kidney disease and the hemoglobin outcomes at the end of the study. There was a positive correlation between higher eGFR at the beginning of the study and reaching higher hemoglobin levels at the end of the study $\left(r_{s}=0.427\right)$. This finding comes in agreement with the finding of Aggarwal et al. (12) where they explained this correlation by stressing the role of uremic toxins in inhibiting erythropoiesis.

In this study, we found no significant difference in changes of eGFR between patients who received ESAs and patients who did not $(\mathrm{P}=0.570$ in the first 4 months and 0.143 in the last 2 months). Our finding comes in agreement with the finding of the study by Koulouridis et al. ${ }^{(13)}$ that found no association between the total study period mean ESAs dose and the GFR change.

\section{CONCLUSION}

We concluded that Intravenous iron supplement is better than oral iron supplement in correction of anemia in pre-dialysis patients. We found that ESAs are effective in raising hemoglobin level in pre-dialysis anemic CKD patients and that ESAs have no adverse effect in the progression of kidney disease.

\section{REFERENCES}

1. Kidney Disease: Improving Global Outcomes (KDIGO) CKD Work Group. (2013): KDIGO 2012 Clinical Practice Guideline for the Evaluation and Management of Chronic Kidney Disease. kdigo.org > wp-content > uploads > 2017/02 > KDIGO_2...

2. Webster A, Nagler E, Morton R et al. (2017): Chronic Kidney Disease. Lancet, 389: 1238-52.

3. Farag Y, Keithi-Reddy S, Mittal B et al. (2011): Anemia, inflammation and health-related quality of life in chronic kidney disease patients. Clinical Nephrology, 75: 524-33.
4. Babitt $\mathbf{J}$, Lin $\mathbf{H}$ (2012): Mechanisms of Anemia in CKD. Journal of the American Society of Nephrology, 23: 1631-34.

5. Shepshelovich D, Rozen-Zvi B, Avni T et al. (2016): Intravenous Versus Oral Iron Supplementation for the Treatment of Anemia in CKD: An Updated Systematic Review and Meta-analysis. In American journal of kidney diseases. The Official Journal of the National Kidney Foundation, 68: 67790.

6. Szczech L, Barnhart H, Inrig J (2008): Secondary analysis of the CHOIR trial epoetin-alpha dose and achieved hemoglobin outcomes. Kidney Int., 74: 791-8.

7. Qunibi W, Martinez C, Smith M (2011): A randomized controlled trial comparing intravenous ferric carboxymaltose with oral iron for treatment of iron deficiency anaemia of nondialysis-dependent chronic kidney disease patients. Nephrol Dial Transplant, 26 (5): 1599-607.

8. Collins A, Kasiske B, Herzog C et al. (2005): Excerpts from the United States Renal Data System 2004 annual data report: atlas of end-stage renal disease in the United States. American Journal of Kidney Diseases, 55: 1-7.

9. Avni T, Bieber A, Grossman A et al. (2015): The safety of intravenous iron preparations: systematic review and metaanalysis. Mayo Clinic Proceedings, 90: 12-23.

10.Wang C, Graham DJ, Kane RC et al. (2015): Comparative Risk of Anaphylactic Reactions Associated With Intravenous Iron Products. Jama., 314: 2062-8.

11. Cody J, Hodson E (2016): Recombinant human erythropoietin versus placebo or no treatment for the anaemia of chronic kidney disease in people not requiring dialysis. https://pubmed.ncbi.nlm.nih.gov/26790135/

12. Aggarwal H, Nand N, Singh S et al. (2003): Comparison of oral versus intravenous iron therapy in predialysis patients of chronic renal failure receiving recombinant human erythropoietin. Journal of the Association of Physicians of India, 51: 170-174.

13. Koulouridis I, Alfayez M, Trikalinos T et al. (2013): Dose of erythropoiesis-stimulating agents and adverse outcomes in CKD: a metaregression analysis. In American journal of kidney diseases. Official Journal of the National Kidney Foundation, 61: 44-56. 